\title{
ANALYTIC DESCRIPTION OF THE ROSSITER-MCLAUGHLIN EFFECT FOR TRANSITING EXOPLANETS: CROSS-CORRELATION METHOD AND COMPARISON WITH SIMULATED DATA
}

\author{
Teruyuki Hirano $^{1}$, Yasushi Suto ${ }^{1,2,3}$, Atsushi Taruya ${ }^{1,2,4}$, Norio Narita ${ }^{5}$, Bun’ei Sato ${ }^{6}$, John Asher Johnson ${ }^{7}$, \\ AND JoshuA N. WINN ${ }^{8}$ \\ ${ }^{1}$ Department of Physics, University of Tokyo, Tokyo 113-0033, Japan; hirano@utap.phys.s.u-tokyo.ac.jp \\ ${ }^{2}$ Research Center for the Early Universe, School of Science, University of Tokyo, Tokyo 113-0033, Japan \\ ${ }^{3}$ Department of Astrophysical Sciences, Princeton University, Princeton, NJ 08544, USA \\ ${ }^{4}$ Institute for the Physics and Mathematics of the Universe (IPMU), University of Tokyo, Chiba 277-8582, Japan \\ ${ }^{5}$ National Astronomical Observatory of Japan, 2-21-1 Osawa, Mitaka, Tokyo 181-8588, Japan \\ ${ }^{6}$ Global Edge Institute, Tokyo Institute of Technology, 2-21-1 Ookayama, Meguro, Tokyo 152-8550, Japan \\ ${ }^{7}$ Department of Astrophysics, California Institute of Technology, MC 249-17 Pasadena, CA 91125, USA \\ ${ }^{8}$ Department of Physics and Kavli Institute for Astrophysics and Space Research, Massachusetts Institute of Technology, Cambridge, MA 02139, USA \\ Received 2009 October 3; accepted 2009 December 2; published 2010 January 4
}

\begin{abstract}
We obtain analytical expressions for the velocity anomaly due to the Rossiter-McLaughlin (RM) effect, for the case when the anomalous radial velocity is obtained by cross-correlation with a stellar template spectrum. In the limit of vanishing width of the stellar absorption lines, our result reduces to the formula derived by Ohta et al., which is based on the first moment of distorted stellar lines. Our new formula contains a term dependent on the stellar line width, which becomes important when rotational line broadening is appreciable. We generate mock transit spectra for four existing exoplanetary systems (HD 17156, TrES-2, TrES-4, and HD 209458) following the procedure of Winn et al., and find that the new formula is in better agreement with the velocity anomaly extracted from the mock data. Thus, our result provides a more reliable analytical description of the velocity anomaly due to the RM effect, and explains the previously observed dependence of the velocity anomaly on the stellar rotation velocity.
\end{abstract}

Key words: planetary systems - planets and satellites: formation - stars: rotation - techniques: radial velocities techniques: spectroscopic

Online-only material: color figures

\section{INTRODUCTION}

Among approximately 400 extrasolar planets discovered as of 2009 September, there are more than 60 transiting planets. The transiting planets provide important information, such as radii and atmospheric signatures, which are unavailable from radial velocity data alone. Another advantage of transiting exoplanets is that one can measure the angle between the stellar rotation axis and the orbital axis of the exoplanet projected onto the sky (conventionally denoted by $\lambda$ ) through the RossiterMcLaughlin (RM) effect. The RM effect generates a radial velocity anomaly during a transit, due to the asymmetric line profiles that result from the partial occultation of the rotating stellar disk (Rossiter et al. 1924; McLaughlin et al. 1924). By carefully investigating velocity anomalies during transits, one can determine the trajectory of planets on the stellar disk and estimate $\lambda$ precisely (e.g., Queloz et al. 2000; Ohta et al. 2005; Winn et al. 2005; Gaudi \& Winn 2007).

This angle is especially important in understanding the basic physical processes of planetary formation and subsequent orbital migration. According to recent planetary formation theories, gaseous planets orbiting within $0.1 \mathrm{AU}$ of the parent star (hot Jupiters) are supposed to have formed a few $\mathrm{AU}$ away from the star, and subsequently to have migrated inward (Ida \& Lin 2004). While standard migration mechanisms keep $\lambda \sim 0^{\circ}$, scenarios such as planet-planet scattering may change $\lambda$ significantly (Lin et al. 1996; Chatterjee et al. 2008; Nagasawa et al. 2008). The Kozai mechanism may also lead to a highly inclined orbit (Wu et al. 2007). Thus, the observed distribution of the angles provides an observational clue to distinguish between different planet formation and migration theories.
Ohta et al. (2005) derived an analytic formula (hereafter, the OTS formula) for velocity anomalies due to the RM effect, by computing the first moment (intensity-weighted mean wavelength) of distorted absorption lines perturbatively. (Previously, Kopal et al. (1942) and others used the first-moment method for eclipsing binary stars to estimate the velocity anomalies.) The OTS formula proved to be useful in understanding the parameter dependence of the velocity anomaly and also in forecasting the error budget of the parameter estimate, in particular of the spin-orbit misalignment angle $\lambda$. Indeed, their work inspired Winn et al. (2005) to revisit the estimate of $\lambda$ precisely for the first discovered transiting planetary system HD 209458. For that purpose, Winn et al. (2005) generated in-transit stellar spectra of HD 209458, put them into the Keck analysis routine of radial velocities, and found that the OTS formula systematically under-predicts the velocity anomaly by $10 \%$. Intriguingly, however, Johnson et al. (2008) and Winn et al. (2008), found the OTS formula to provide an adequate description of the simulated results for the cases of HAT-P-1 and TrES-2.

We noted that the systematic difference between the OTS formula and the simulation may be sensitive to the stellar spin rotation velocity $v \sin i_{\star}\left(i_{\star}\right.$ denotes the inclination angle of the stellar spin axis), which is $4.70 \pm 0.16 \mathrm{~km} \mathrm{~s}^{-1}, 3.75 \pm 0.58 \mathrm{~km} \mathrm{~s}^{-1}$, and $1.0 \pm 0.6 \mathrm{~km} \mathrm{~s}^{-1}$, for the HD 209458, HAT-P-1, and TrES-2 systems, respectively. In addition, the actual radial velocity measurement algorithm does not adopt the moment method, strictly speaking. For instance, the analysis routines used by the High Accuracy Radial velocity Planet Searcher (HARPS) and Spectrographe pour l'Observation des Phénomènes des Intérieurs stellaires et des Exoplanètes (SOPHIE) teams involve the crosscorrelation of the observed spectrum with a template spectrum, 


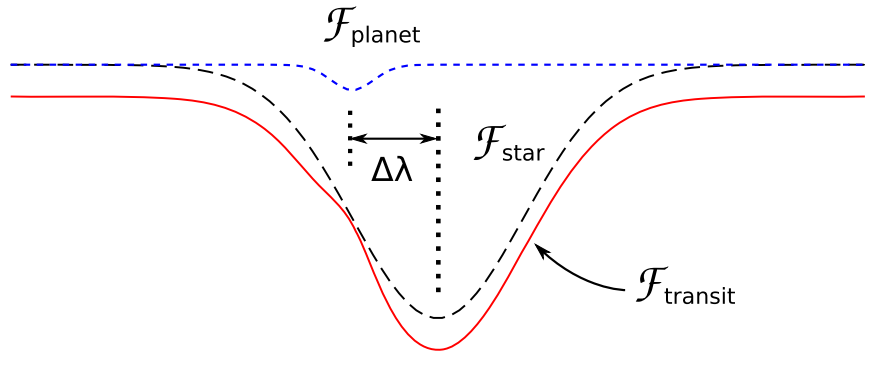

wavelength

Figure 1. Schematic absorption line profiles of a transiting planetary system: $\mathcal{F}_{\text {star }}(\lambda)$ is a symmetric line profile with respect to the central wavelength of each line $\lambda_{0}$ over the entire stellar disk outside the transit, while $\mathcal{F}_{\text {planet }}(\lambda-\Delta \lambda)$ is a symmetric line profile for the portion occulted by a planet that is shifted by a wavelength of $\Delta \lambda$ with respect to $\lambda_{0}$. The resulting distorted stellar line profile in transit is given by $\mathcal{F}_{\text {transit }}(\lambda)=\mathcal{F}_{\text {star }}(\lambda)-\mathcal{F}_{\text {planet }}(\lambda-\Delta \lambda)$.

(A color version of this figure is available in the online journal.)

and the velocity anomaly is estimated from the peak of the cross-correlation function. The analysis routine which uses the iodine cell technique, with the Subaru High Dispersion Spectrograph (HDS) or Keck High Resolution Echelle Spectrometer (HIRES), involves a fit to the observed spectra, based on a template spectrum and the known spectrum of the iodine cell.

For these reasons, in this paper we revisit the analytic approach to the RM effect using the cross-correlation method. Our analytic approach to this problem is complementary to the recent numerical approach by Triaud et al. (2009), and provides an analytic framework for understanding their results. Even though the cross-correlation method is not directly applicable to the analysis of the iodine cell technique, we find that our result seems to capture the main qualitative features of the numerical results based on that technique; specifically, our formula includes a term dependent on the stellar spin velocity that does not show up in the moment method but was empirically found by Winn et al. (2005).

The rest of the paper is organized as follows. Section 2 describes our analytical modeling of stellar absorption lines distorted by the occultation due to a transiting planet (Section 2.1) and derives the RM velocity anomaly on the basis of the moment method (Section 2.2) and the cross-correlation method (Section 2.3). In order to check the reliability of the new analytic formula, we generate simulated spectra during transits and put them into the Subaru analysis routine of radial velocities in Section 3 as Winn et al. (2005) did for the Keck routine. Specifically, we consider HD $17156\left(v \sin i_{\star}=4.2 \mathrm{~km} \mathrm{~s}^{-1}\right)$, TrES-2 $\left(v \sin i_{\star}=1.0 \mathrm{~km} \mathrm{~s}^{-1}\right)$, TrES-4 $\left(v \sin i_{\star}=8.5 \mathrm{~km} \mathrm{~s}^{-1}\right)$, and HD $209458\left(v \sin i_{\star}=4.5 \mathrm{~km} \mathrm{~s}^{-1}\right)$ systems, and find that our new formula reproduces the simulated data better than the OTS formula. Finally, Section 4 is devoted to a summary and further discussion of the present paper.

\section{ANALYTIC MODEL FOR THE VELOCITY ANOMALY}

In this section, we analytically derive the velocity anomaly due to the RM effect based on the two different methods. The final result for the moment method is Equation (16). For the cross-correlation method, the key result is Equation (37) when the stellar line profile and the rotational broadening kernel are approximated by Equations (20) and (22), respectively. Readers who are not interested in the mathematical details of

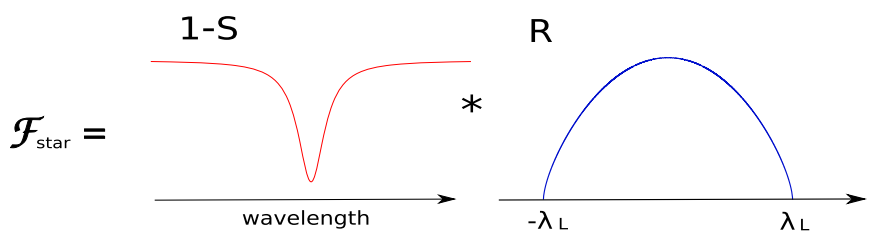

Figure 2. Stellar absorption line profile $\mathcal{F}_{\text {star }}$, which is a convolution of the intrinsic stellar line profile (including the natural broadening), $S(\lambda)$, and the stellar rotational kernel $R\left(\lambda ; \lambda_{L}\right)$.

(A color version of this figure is available in the online journal.)

the derivations may simply skip this section and directly move to Section 3.

\subsection{Velocity Anomaly During a Transit}

The stellar absorption line profile distorted by the RM effect is schematically illustrated in Figure 1. During a transit, a portion on the stellar disk is occulted by a planet, and the flux contribution from the portion occulted by the planet, $\mathcal{F}_{\text {planet }}(\lambda-\Delta \lambda)$ is subtracted from the stellar absorption line, $\mathcal{F}_{\text {star }}(\lambda)$, yielding a distorted absorption line profile in transit, $\mathcal{F}_{\text {transit }}(\lambda)$. The center of $\mathcal{F}_{\text {planet }}$ is Doppler-shifted by $\Delta \lambda$ relative to the central wavelength $\lambda_{0}$ of $\mathcal{F}_{\text {star }}$ since the portion occulted by the planet has a stellar rotation velocity,

$$
v_{p}=c \frac{\Delta \lambda}{\lambda_{0}},
$$

along the line of sight of an observer. Because of the distortion, the absorption line profile becomes asymmetric, producing an apparent velocity anomaly when the radial velocity is estimated using one of the standard techniques.

Let us first write a stellar absorption line profile as a convolution of an intrinsic line profile $S(\lambda)$ and a stellar rotation kernel $R\left(\lambda ; \lambda_{L}\right)$ as

$$
\mathcal{F}_{\text {star }}(\lambda)=1-S(\lambda) * R\left(\lambda ; \lambda_{L}\right),
$$

where the symbol $*$ indicates a convolution, and the continuum level is normalized to unity (Figure 2). In this section, we implicitly focus on a particular single line of a central wavelength $\lambda_{0}$, and locate the center of $S(\lambda)$ at $\lambda_{0}$. Then we normalize $S(\lambda)$ and $R\left(\lambda ; \lambda_{L}\right)$ so that

$$
\int_{-\infty}^{\infty} S(\lambda) d \lambda=1, \quad \int_{-\infty}^{\infty} R\left(\lambda ; \lambda_{L}\right) d \lambda=1
$$

A Voigt profile (convolution of a Gaussian and a Lorentzian; see Equation (19)) is often used to approximate $S(\lambda)$, but a Gaussian profile also provides a reasonable approximation. We will mainly be concerned with the Gaussian approximation in Section 2.3.

The function $R\left(\lambda ; \lambda_{L}\right)$ indicates a rotational broadening kernel and broadens the intrinsic stellar line at $\lambda_{0}$ over the range of $\lambda_{0}-\lambda_{L}$ and $\lambda_{0}+\lambda_{L}$, where $\lambda_{L} / \lambda_{0}=v \sin i_{\star} / c$. Its expression for a rigid rotating stellar disk can be computed once the limb-darkening law is specified (see, e.g., Gray 2005). In this paper, we consider the quadratic limb-darkening law in which the intensity of the stellar disk at a position $(x, y)$ relative to the center of the star is expressed as

$$
\begin{aligned}
\frac{I(x, y)}{I_{c}} & =1-u_{1}(1-\cos \theta)-u_{2}(1-\cos \theta)^{2}, \\
\cos \theta & =\sqrt{1-\frac{x^{2}+y^{2}}{R_{s}^{2}}} .
\end{aligned}
$$


In the above expression, $\theta$ is the angle between the line of sight and the normal vector to the local stellar surface, $R_{S}$ is the stellar radius, the coefficients $u_{1}$ and $u_{2}$ are the limb-darkening parameters, and $I_{c}$ indicates the intensity at the center of the stellar disk.

Then we obtain

$$
\begin{aligned}
R\left(\lambda ; \lambda_{L}\right)= & c_{1} \sqrt{1-\left(\frac{\lambda}{\lambda_{L}}\right)^{2}}+c_{2}\left\{1-\left(\frac{\lambda}{\lambda_{L}}\right)^{2}\right\} \\
& +c_{3}\left\{1-\left(\frac{\lambda}{\lambda_{L}}\right)^{2}\right\}^{3 / 2},
\end{aligned}
$$

where the coefficients $c_{1}, c_{2}$, and $c_{3}$ are given as

$$
\begin{gathered}
c_{1}=\frac{2\left(1-u_{1}-u_{2}\right)}{\pi \lambda_{L}\left(1-u_{1} / 3-u_{2} / 6\right)}, \\
c_{2}=\frac{u_{1}+2 u_{2}}{2 \lambda_{L}\left(1-u_{1} / 3-u_{2} / 6\right)}, \\
c_{3}=-\frac{4 u_{2}}{3 \pi \lambda_{L}\left(1-u_{1} / 3-u_{2} / 6\right)} .
\end{gathered}
$$

Adopting Equation (2) as a line profile, the line distortion due to the RM effect is described as follows. In the transiting planetary system, the portion occulted by the planet is sufficiently small. Thus the effect of the rotational broadening within the portion is safely negligible, and one can write

$$
\mathcal{F}_{\text {planet }}(\lambda-\Delta \lambda)=f\{1-S(\lambda-\Delta \lambda)\},
$$

where $f$ is the ratio of the flux from the occulted part of the stellar disk to the total flux.

In general, $\Delta \lambda=v_{p} \lambda_{0} / c$ is a function of the position of the planet, and the contribution of wavelength shift is determined by integrating over the entire occulted portion of stellar disk. The precise definition of $v_{p}$ in terms of the stellar rotation velocity and the position of the planet is given by Equation (A7), which is simplified as Equation (A8) for rigid rotation.

Then, the line profile during a transit is given as

$$
\begin{aligned}
\mathcal{F}_{\text {transit }}(\lambda) & =\mathcal{F}_{\text {star }}(\lambda)-\mathcal{F}_{\text {planet }}(\lambda-\Delta \lambda) \\
& =\left\{1-S(\lambda) * R\left(\lambda ; \lambda_{L}\right)\right\}-f\{1-S(\lambda-\Delta \lambda)\} .
\end{aligned}
$$

Because the continuum level $1-f$ in Equation (10) does not change the evaluation of the velocity anomaly, in what follows we simply use the expression

$$
\mathcal{F}_{\text {transit }}(\lambda)=-S(\lambda) * R\left(\lambda ; \lambda_{L}\right)+f S(\lambda-\Delta \lambda),
$$

without losing generality.

\subsection{Estimate Based on the Moment Method}

Let us consider first the moment method, in which we estimate the wavelength shift of the center of a distorted line during a transit as (e.g., Ohta et al. 2005)

$$
\delta_{\mathrm{RM}} \equiv \frac{\int_{-\infty}^{\infty} \lambda \mathcal{F}_{\text {transit }}(\lambda) d \lambda}{\int_{-\infty}^{\infty} \mathcal{F}_{\text {transit }}(\lambda) d \lambda} .
$$

Substituting Equation (11) into $\mathcal{F}_{\text {transit }}(\lambda)$ in Equation (12), the denominator becomes

$$
\begin{aligned}
\int_{-\infty}^{\infty} \mathcal{F}_{\text {transit }}(\lambda) d \lambda= & -\int_{-\infty}^{\infty} d \lambda^{\prime} R\left(\lambda^{\prime} ; \lambda_{L}\right) \int_{-\infty}^{\infty} d \lambda S\left(\lambda-\lambda^{\prime}\right) \\
& +f \int_{-\infty}^{\infty} d \lambda S(\lambda-\Delta \lambda) \\
= & -1+f .
\end{aligned}
$$

The numerator of Equation (12) reduces to

$$
\begin{aligned}
\int_{-\infty}^{\infty} \lambda & \mathcal{F}_{\text {transit }}(\lambda) d \lambda=-\int_{-\infty}^{\infty} d \lambda^{\prime} R\left(\lambda^{\prime} ; \lambda_{L}\right) \int_{-\infty}^{\infty} \lambda S\left(\lambda-\lambda^{\prime}\right) d \lambda \\
& +f \int_{-\infty}^{\infty} \lambda S(\lambda-\Delta \lambda) d \lambda \\
= & -\int_{-\infty}^{\infty} R\left(\lambda^{\prime} ; \lambda_{L}\right) d \lambda^{\prime} \int_{-\infty}^{\infty}\left(\lambda-\lambda^{\prime}\right) S\left(\lambda-\lambda^{\prime}\right) d \lambda \\
& -\int_{-\infty}^{\infty} \lambda^{\prime} R\left(\lambda^{\prime} ; \lambda_{L}\right) d \lambda^{\prime} \int_{-\infty}^{\infty} S\left(\lambda-\lambda^{\prime}\right) d \lambda \\
& +f \int_{-\infty}^{\infty}(\lambda-\Delta \lambda) S(\lambda-\Delta \lambda) d \lambda \\
& +f \int_{-\infty}^{\infty} \Delta \lambda S(\lambda-\Delta \lambda) d \lambda \\
= & f \int_{-\infty}^{\infty} \Delta \lambda S(\lambda-\Delta \lambda) d \lambda \\
= & f \Delta \lambda
\end{aligned}
$$

and therefore we obtain

$$
\delta_{\mathrm{RM}}=-\frac{f}{1-f} \Delta \lambda .
$$

Using Equation (1), one can rewrite Equation (15) in terms of the velocity anomaly as

$$
\Delta v \equiv c \frac{\delta_{\mathrm{RM}}}{\lambda_{0}}=-\frac{f}{1-f} v_{p} .
$$

When we explicitly write the flux ratio $f$ and the subplanet velocity $v_{p}$ as a function of the planet position, we reproduce the OTS formula (see Appendices A and B). In particular, the above expression coincides with Equation (25) of OTS which is the formula for a complete transiting case without limb darkening, if we simply replace the quantities of $f$ and $v_{p}$ with $\gamma^{2}$ and $\Omega_{s} x \sin i_{\star}$ (Ohta et al. 2009).

Note that Equations (13)-(15) are derived from the normalization condition (3) of $S(\lambda)$ and $R\left(\lambda ; \lambda_{L}\right)$ and the fact that they are even functions of $\lambda$, and do not depend on their specific functional forms. This implies that the velocity anomaly derived from the moment method depends neither on the profile nor on the width of the absorption line. Thus the results are independent of the stellar rotation velocity.

\subsection{Estimate Based on the Cross-correlation Method}

\subsubsection{Formulation for the Voigt Profile}

Consider next the estimate of the velocity anomaly based on the cross-correlation method. In this case, the wavelength shift of central line $\delta_{\mathrm{RM}}$ is obtained by maximizing the cross-correlation function:

$$
\left.\frac{d C(x)}{d x}\right|_{x=\delta_{\mathrm{RM}}}=0,
$$




$$
C(x)=\int_{-\infty}^{\infty} \mathcal{F}_{\text {star }}(\lambda-x) \mathcal{F}_{\text {transit }}(\lambda) d \lambda .
$$

To proceed further, we have to specify the functional form of $S(\lambda)$ and $R\left(\lambda, \lambda_{L}\right)$.

Here we adopt the Voigt profile for the stellar line:

$$
\begin{gathered}
S(\lambda)=G_{\mathrm{S}}(\lambda ; \beta) * L(\lambda ; \gamma), \\
G_{\mathrm{S}}(\lambda ; \beta)=\frac{1}{\beta \sqrt{\pi}} e^{-\lambda^{2} / \beta^{2}}, \\
L\left(\lambda ; \gamma_{\star}\right)=\frac{1}{\pi} \frac{\gamma_{\star}}{\lambda^{2}+\gamma_{\star}^{2}} .
\end{gathered}
$$

In the above expressions, $\beta$ in the Gaussian kernel characterizes the thermal and microturbulent broadening, while $\gamma_{\star}$ in the Lorentzian kernel represents the line broadening due to natural broadening and the Stark effect.

While we derive Equation (5) for the rotational kernel $R\left(\lambda ; \lambda_{L}\right)$ for the quadratic limb-darkening law, the expression is not analytically tractable. Thus we approximate it by a Gaussian:

$$
R\left(\lambda ; \lambda_{L}\right) \approx G_{\mathrm{R}}(\lambda ; \sigma) \equiv \frac{1}{\sigma \sqrt{\pi}} e^{-\lambda^{2} / \sigma^{2}},
$$

where the relation between $\sigma$ and $\lambda_{L}$ is obtained by least-squares fitting of Gaussian to Equation (5). Their explicit relation is given in Appendix F.

Under this Gaussian approximation for the rotation kernel, we have

$$
\begin{aligned}
S(\lambda) * R\left(\lambda ; \lambda_{L}\right) & \approx G_{\mathrm{S}}(\lambda ; \beta) * L\left(\lambda ; \gamma_{\star}\right) * G_{\mathrm{R}}(\lambda ; \sigma) \\
& \equiv G\left(\lambda ; \beta_{\star}\right) * L\left(\lambda ; \gamma_{\star}\right),
\end{aligned}
$$

where

$$
\beta_{\star}^{2} \equiv \beta^{2}+\sigma^{2}
$$

This significantly simplifies the analytic computation, and we now have

$$
\begin{aligned}
& \mathcal{F}_{\text {transit }}(\lambda)=-\int_{-\infty}^{\infty} G\left(\lambda^{\prime} ; \beta_{\star}\right) L\left(\lambda-\lambda^{\prime} ; \gamma_{\star}\right) d \lambda^{\prime} \\
&+f \int_{-\infty}^{\infty} G\left(\lambda^{\prime} ; \beta_{p}\right) L\left(\lambda-\Delta \lambda-\lambda^{\prime} ; \gamma_{p}\right) d \lambda^{\prime} \\
& \mathcal{F}_{\text {star }}(\lambda)=-\int_{-\infty}^{\infty} G\left(\lambda^{\prime} ; \beta_{\star}\right) L\left(\lambda-\lambda^{\prime} ; \gamma_{\star}\right) d \lambda^{\prime}
\end{aligned}
$$

where $\beta_{p}$ and $\gamma_{p}$ indicate the narrow line originated from the portion of the planet, and thus we simply set $\beta_{p}=\beta$.

Substituting Equations (25) and (26) into Equations (17) and (18), we obtain the following algebraic equation for $\delta_{\mathrm{RM}}$ :

$$
\begin{aligned}
\frac{1}{b^{3 / 2}} e^{-\left(a^{2}-c^{2}\right) / 4 b} \operatorname{Re}\left\{(a-i c) e^{i a c / 2 b}\left[1+\operatorname{erfi}\left(\frac{a-i c}{2 \sqrt{b}}\right)\right]\right\} \\
=\frac{f}{B^{3 / 2}} e^{-\left(A^{2}-C^{2}\right) / 4 B} \operatorname{Re}\left\{(A-i C) e^{i A C / 2 B}\right. \\
\left.\quad \times\left[1+\operatorname{erfi}\left(\frac{A-i C}{2 \sqrt{B}}\right)\right]\right\},
\end{aligned}
$$

where

$$
a=2 \pi \delta_{\mathrm{RM}}, b=2 \pi^{2} \beta_{\star}^{2}, c=4 \pi \gamma_{\star},
$$

$$
A=2 \pi\left(\delta_{\mathrm{RM}}-\Delta \lambda\right), B=\pi^{2}\left(\beta_{\star}^{2}+\beta_{p}^{2}\right), C=2 \pi\left(\gamma_{\star}+\gamma_{p}\right),
$$

and $\operatorname{erfi}(z)$ denotes the imaginary error function defined in terms of the error function $\operatorname{erf}(x)$ :

$$
\operatorname{erfi}(z) \equiv-i \operatorname{erf}(i z)=\frac{2}{i \sqrt{\pi}} \int_{0}^{i z} e^{-t^{2}} d t
$$

The derivation of Equation (27) is summarized in Appendix C.

\subsubsection{Analytic Formula for the Gaussian Profile}

Since the Voigt profile is widely used for stellar absorption lines, Equation (27) is fairly general. While it cannot be solved exactly for $\delta_{\mathrm{RM}}$, we obtain an approximate perturbation solution for $\delta_{\mathrm{RM}} \ll \Delta \lambda$. In particular, we present the result for the Gaussian profile $\left(\gamma_{\star}=\gamma_{p}=0\right)$ in this subsection, which will be compared with simulated data analysis in Section 3. The other solutions for the Lorentzian profile $\left(\beta_{\star}=0\right)$ and the Gaussian profile with a small Lorentzian wing $\left(\gamma_{\star} \ll \beta_{\star}\right)$ are presented in Appendices D and E, respectively.

In the Gaussian profile assumed here, the parameters $c$ and $C$ in Equations (28) and (29) vanish. Then Equation (27) reduces to

$$
\frac{a}{b^{3 / 2}} e^{-a / 4 b}=f \frac{A}{B^{3 / 2}} e^{-A / 4 B},
$$

or equivalently

$$
f=\left(\frac{\beta_{\star}^{2}+\beta_{p}^{2}}{2 \beta_{\star}^{2}}\right)^{3 / 2} \frac{\delta_{\mathrm{RM}}}{\delta_{\mathrm{RM}}-\Delta \lambda} \exp \left\{-\frac{\delta_{\mathrm{RM}}^{2}}{2 \beta_{\star}^{2}}+\frac{\left(\delta_{\mathrm{RM}}-\Delta \lambda\right)^{2}}{\beta_{\star}^{2}+\beta_{p}^{2}}\right\} .
$$

Note that $f$ is approximately the square of the planet-tostar radius ratio, and is of order $10^{-2}$ for giant planets. Thus, Equation (15) implies that $\left|\delta_{\mathrm{RM}} / \Delta \lambda\right| \approx f \ll 1$, and we can expand the exponent in Equation (32) up to the linear order of $\delta_{\mathrm{RM}}$ :

$$
\begin{aligned}
-\frac{\delta_{\mathrm{RM}}^{2}}{2 \beta_{\star}^{2}}+\frac{\left(\delta_{\mathrm{RM}}-\Delta \lambda\right)^{2}}{\beta_{\star}^{2}+\beta_{p}^{2}} & \approx \frac{\Delta \lambda^{2}}{\beta_{\star}^{2}+\beta_{p}^{2}}\left(1-2 \frac{\delta_{\mathrm{RM}}}{\Delta \lambda}\right) \\
& \equiv \kappa\left(1-2 \frac{\delta_{\mathrm{RM}}}{\Delta \lambda}\right) .
\end{aligned}
$$

In general, we consider the case where $\Delta \lambda$ is well within the width of the stellar broadening, and therefore $\kappa \ll 1$. Therefore, we expand Equation (32) up to the linear order in $\delta_{\mathrm{RM}} / \Delta \lambda$ as

$$
f \approx-\left(\frac{\beta_{\star}^{2}+\beta_{p}^{2}}{2 \beta_{\star}^{2}}\right)^{3 / 2} \frac{\delta_{\mathrm{RM}}}{\Delta \lambda}(1+\kappa),
$$

and finally obtain

$$
\delta_{\mathrm{RM}} \approx-\left(\frac{2 \beta_{\star}^{2}}{\beta_{\star}^{2}+\beta_{p}^{2}}\right)^{3 / 2} f \Delta \lambda\left(1-\frac{\Delta \lambda^{2}}{\beta_{\star}^{2}+\beta_{p}^{2}}\right) .
$$


As before, the above equation can be rewritten as

$$
\Delta v \approx-\left(\frac{2 \beta_{\star}^{2}}{\beta_{\star}^{2}+\beta_{p}^{2}}\right)^{3 / 2} f v_{p}\left\{1-\frac{\lambda_{0}^{2}}{c^{2}\left(\beta_{\star}^{2}+\beta_{p}^{2}\right)} v_{p}^{2}\right\}
$$

or

$$
\Delta v \approx-\left\{\frac{2\left(\beta^{2}+\sigma^{2}\right)}{2 \beta^{2}+\sigma^{2}}\right\}^{3 / 2} f v_{p}\left\{1-\frac{\lambda_{0}^{2}}{c^{2}\left(2 \beta^{2}+\sigma^{2}\right)} v_{p}^{2}\right\},
$$

where we replace $\beta_{p}^{2}$ with $\beta^{2}$ (line width without rotational broadening), and $\beta_{\star}^{2}$ with $\beta^{2}+\sigma^{2}$ in which $\sigma$ indicates the stellar rotation width.

Equation (37) is the key result of the present paper which describes the RM velocity anomaly under the Gaussian approximation for the intrinsic line profile and the stellar rotation kernel. In marked contrast to Equation (16) based on the moment method, Equation (37) indicates that the radial velocity anomaly from the cross-correlation method depends both on the width of the line profile $(\beta)$ and on the rotation velocity $(\sigma)$. It recovers the OTS formula only when $\sigma=0$ and the second term in the parentheses is negligibly small.

Indeed, the presence of the cubic term in $v_{p}^{3}$ is consistent with the empirical finding from the simulated data (Winn et al. 2005). Note that a quintic (fifth-order term) was needed to match the simulations for the rapidly rotating star HAT-P-2 (Winn et al. 2007), which would emerge naturally in our formulation by expanding the next-order term in $\kappa$. We should note here that in many cases the estimate of the spin-orbit misalignment angle $\lambda$ is not very sensitive to the choice of the moment method or the cross-correlation method discussed in this section; when $\lambda$ is small and the transit impact parameter is not too close to zero, then $\lambda$ is mainly determined by the epoch of the photometric central transit when $\Delta v$, and thus $v_{p}$, vanish.

\section{COMPARISON WITH SIMULATED RESULTS BASED ON THE SUBARU ANALYSIS ROUTINE}

So far, we have focused on the RM velocity anomaly estimated from single line profiles characterized by the Voigt function. The practical data analysis, however, is much more complicated; the wavelength shift $\Delta \lambda$ is estimated statistically from many different lines, and each line is not always approximated by a single Voigt profile. In addition, Subaru and Keck analysis routines are not related in a straightforward way to the crosscorrelation algorithm. This is why we create mock spectrum data during the transit, compute radial velocity anomalies using the Subaru analysis routine, and compare the output results with our analytic formulae, in particular Equation (37).

\subsection{Subaru Analysis Routine}

In order to check the validity of our analytic formula, we create mock spectra in a transit, and reduce the data with the analysis routine (Sato et al. 2002) for the Subaru HDS radial velocity measurement with the $\mathrm{I}_{2}$ cell. The routine has been extensively used for the previous observation of the RM effect (e.g., Narita et al. 2007, 2008).

The routine finds the best-fit value of the wavelength shift $\delta \lambda$ by modeling an observed spectrum $I_{\text {obs }}(\lambda)$ with the given transmission function of the $\mathrm{I}_{2}$ cell, $A(\lambda)$, as

$$
I_{\mathrm{obs}}(\lambda)=k[A(\lambda) S(\lambda-\delta \lambda)] * \mathrm{IP}
$$

where $k$ is the normalization factor, $S(\lambda-\delta \lambda)$ is the intrinsic stellar spectrum shifted by $\delta \lambda$, and IP denotes the instrumental profile. Sato et al. (2002) used the Lick-Hamilton $I_{2}$ cell spectrum for $A(\lambda)$ with high spectral resolution $(R \sim 400,000)$. The peculiar velocity and the Keplerian motion of the star as well as the RM velocity anomaly contribute to $\delta \lambda$, and thus the former two have to be subtracted in order to extract $\delta_{\mathrm{RM}}$ alone.

Once $A(\lambda)$ and $S(\lambda)$ are given, the routine simultaneously finds the best-fit values for $k, \delta \lambda$, parameters characterizing IP (usually expressed as a linear combination of several Gaussians), and calibration parameters of wavelength by least-squares fit to $I_{\text {obs }}(\lambda)$. In reality, the method of Sato et al. (2002) attempts to elaborate the intrinsic stellar template spectrum $S(\lambda)$ as well from the observed spectrum $I_{\mathrm{obs}}(\lambda)$. In practice, it proceeds as follows:

1. Choose an initial stellar spectrum $S^{0}(\lambda)$ from an observational data set or from a theoretical model.

2. Find the best-fit parameters in the right-hand side of Equation (38), and denote the resultant best-fit spectrum by $I^{0}(\lambda)$.

3. Compute the residual:

$$
\delta S(\lambda)=I_{\mathrm{obs}}(\lambda)-I^{0}(\lambda)
$$

and use

$$
S^{1}(\lambda)=S^{0}(\lambda)+\delta S(\lambda)
$$

as a revised template for the stellar spectrum.

4. Iterate the above steps until the given accuracy is achieved.

In general, the above method finds the best-fit stellar template spectrum separately for each $I_{\mathrm{obs}}(\lambda)$ observed at different epochs. In order to avoid the contamination caused by the $\mathrm{I}_{2}$ absorption lines in using only one spectrum, the set of stellar templates at different times are averaged and the resulting stellar spectrum $\bar{S}(\lambda)$ is used to determine $\delta \lambda$ for each $I_{\mathrm{obs}}(\lambda)$.

The Keck HIRES analysis routine by Butler et al. (1996) uses the stellar template spectrum directly obtained from the observed spectrum (without the $\mathrm{I}_{2}$ cell) by deconvolving the instrumental profile that is estimated based on observations of a rapidly rotating $\mathrm{B}$ star using the $\mathrm{I}_{2}$ cell. Except for the treatment of the stellar spectrum, both routines are very similar, and our simulation result below is applicable to the Keck data analysis.

\subsection{Simulated Spectra}

As mock observational spectra for the Subaru analysis routine $\left(I_{\text {obs }}(\lambda)\right.$ in Equation (38)), we create many simulated spectra during transits following the procedure of Winn et al. (2005) as follows:

1. Broaden the National Solar Observatory (NSO) solar spectrum (Kurucz et al. 1984) so as to the include the effect of stellar rotation. ${ }^{9}$ We apply two different broadening kernels as discussed below.

2. Compute the spectra during the transit from Equation (10) using the broadened spectrum described above for $\mathcal{F}_{\text {star }}(\lambda)$, and the original (unbroadened) spectrum for $\mathcal{F}_{\text {planet }}(\lambda-\Delta \lambda)$.

3. Multiply $\mathcal{F}_{\text {transit }}(\lambda)$ by $A(\lambda)$, the transmission function of the $\mathrm{I}_{2}$ cell (Equation (38)).

\footnotetext{
9 Although the NSO solar spectrum is a rotationally broadened spectrum whose rotational velocity $v \sin i_{\star} \simeq 1.85 \mathrm{~km} \mathrm{~s}^{-1}$, we additionally broaden the spectrum by Gaussian broadening kernels.
} 


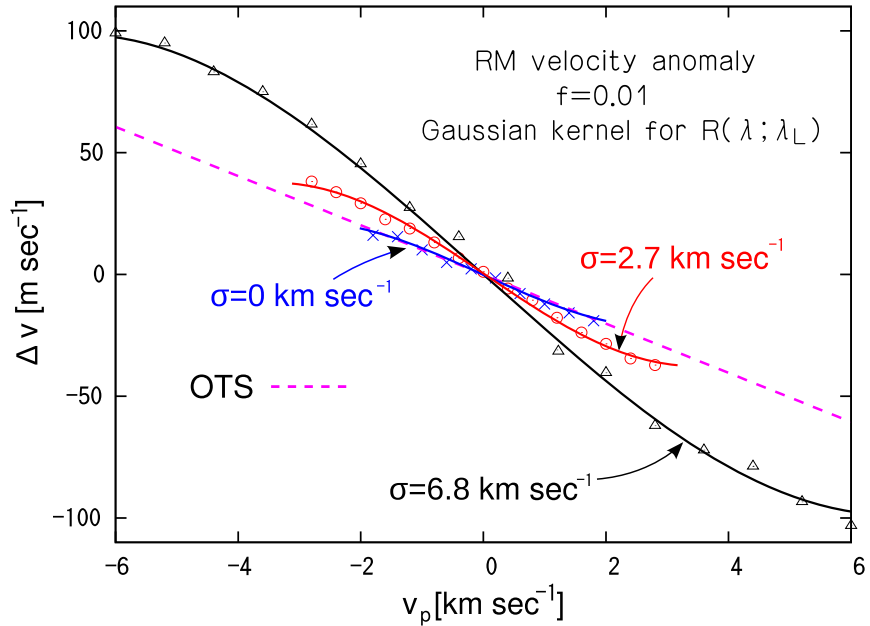

Figure 3. RM velocity anomaly against $v_{p}$ for $f=0.01$. Symbols: results extracted from mock data using the Subaru analysis routine with NSO spectrum for $S(\lambda)$ and the Gaussian broadening kernel (Equation (22)). Crosses, circles, and triangles correspond to $\sigma=0,2.7$, and $6.8 \mathrm{~km} \mathrm{~s}^{-1}$, respectively. The number of data points shown is suppressed to make it easier to see. Solid lines: polynomial fit (Equation (41)) to the mock results. The OTS formula is plotted in a dashed line for comparison (almost indistinguishable from the $\sigma=0$ line). (A color version of this figure is available in the online journal.)

4. Convolve $\mathcal{F}_{\text {transit }}(\lambda) A(\lambda)$ with the instrumental profile, IP, which is approximated by a single Gaussian that best fits the IP of Subaru/HDS. We call the resultant spectra "the mock data."

5. Feed the mock spectra into the Subaru analysis routine (Section 3.1), and compute $\delta \lambda$.

For the broadening kernel for the stellar rotation in step (1), we first adopt a Gaussian (Section 3.3.1) so as to compare the analytic formula under the Gaussian approximation, and then use Equation (5) for more realistic comparison.

Once the broadening kernel is specified, the remaining parameters are the flux ratio $f$ and the subplanet velocity $v_{p}=c \Delta \lambda / \lambda$. We repeat the above procedure at grids on the $\left(f, v_{p}\right)$ plane. We select $f=0.00,0.004,0.008,0.010,0.012,0.016,0.020$, and assign to $v_{p}$ typically 20-30 different values ranging over several $\mathrm{km} \mathrm{s}^{-1}$ for each $f$ in equal interval. Then we compute the velocity anomaly $\Delta v$ on those grids of $\left(f, v_{p}\right)$.

Finally, we apply the methodology to four existing planetary systems (HD 17156, TrES-2, TrES-4, and HD 209458), and fit the result to the following form inspired by the Gaussian approximation (Equation (37)):

$$
\Delta v=-f v_{p}\left(p-q v_{p}^{2}\right),
$$

where $p$ and $q$ are constants fitted for each planetary system, and depend on the stellar absorption line profile and rotation broadening among others.

\subsection{Results}

\subsubsection{Ideal Case with the Gaussian Rotational Kernel}

Consider the NSO spectrum additionally broadened with the Gaussian kernel (Equation (22)). We adopt $\sigma=0,2.7$, and $6.8 \mathrm{~km} \mathrm{~s}^{-1},{ }^{10}$ create mock spectra during transits for grids

\footnotetext{
10 The dispersion parameter $\sigma$ (or $\beta$ ) has a unit of length, but we here express them in terms of radial velocities in order to make it easier to compare the dispersion parameters with the stellar rotational velocities.
}

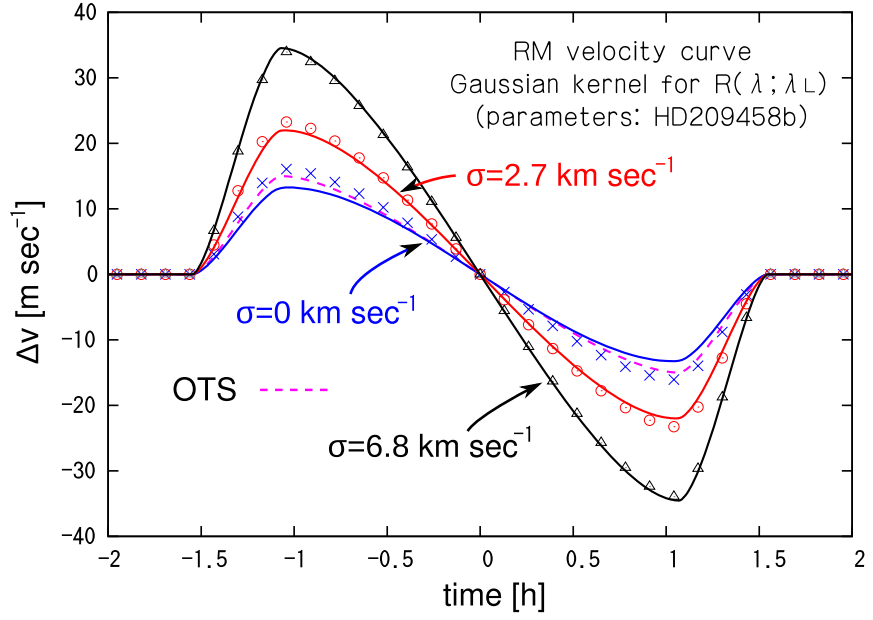

Figure 4. RM velocity anomaly curve for HD 209458 but with varying $\sigma$ for the Gaussian broadening kernel. The central transit time is set to the origin of the time. The spin-orbit misalignment angle $\lambda$ is assumed to be $0^{\circ}$. Symbols: predictions based on the polynomial fit (Equation (41)) plotted in Figure 3. Crosses, circles, and triangles correspond to $\sigma=0,2.7$, and $6.8 \mathrm{~km} \mathrm{~s}^{-1}$, respectively. Solid lines: analytic formula (Equation (37)) with the Gaussian broadening kernel based on the cross-correlation method. The OTS formula is plotted in a dashed line for comparison.

(A color version of this figure is available in the online journal.)

on the $\left(f, v_{p}\right)$ plane, and determine the wavelength shift $\Delta \lambda$. The velocity anomaly $\Delta v$ against $v_{p}$ is plotted in Figure 3 for $f=0.01$. Since $\Delta v$ is simply proportional to $f$, this plot can be easily scaled for an arbitrary value of $f$ as long as $f \ll 1$.

Different absorption lines in the NSO spectrum have different line profiles and are not exactly Gaussian. If the Gaussian approximation (Equation (37)) were exact, the coefficients in Equation (41) for a particular line at wavelength $\lambda_{0}$ should be given as

$p=\left[1+\frac{\sigma^{2}}{2 \beta^{2}+\sigma^{2}}\right]^{3 / 2}, \quad q=\left[1+\frac{\sigma^{2}}{2 \beta^{2}+\sigma^{2}}\right]^{3 / 2} \frac{\lambda_{0}^{2}}{c^{2}\left(2 \beta^{2}+\sigma^{2}\right)}$.

Apparently, they should be sensitive to each absorption line profile (its central wavelength $\lambda_{0}$ and width $\beta$ ), and it is not clear that the real data analysis based on many different lines overall reproduces the polynomial form like Equation (41). Nevertheless, Figure 3 indicates that the simulated results are indeed fitted very well by Equation (41). As expected, the OTS formula derived from the moment method agrees with the simulated result if the stellar rotational broadening is negligible $(\sigma=0)$.

Given the above encouraging result, we can use Equation (41) to compute the velocity anomaly curve. In order to do so, we have to compute $f$ and $v_{p}$ as a function of time, which can be done using the formulae in Appendices A and B (see also OTS). Figure 4 plots the prediction for HD 209458 in symbols on the basis of the result plotted in Figure 3. Also plotted for comparison is the analytic formula (37) for the Gaussian kernel adopting $\beta=2.6 \mathrm{~km} \mathrm{~s}^{-1}$ as the best fit of the original solar spectrum. We here adopt $\lambda_{0}=5500 \AA$ as a typical wavelength of the radial velocity analysis. ${ }^{11}$ Since the actual stellar rotation speed of HD 209458 is $v \sin i_{\star}=4.70 \pm 0.16 \mathrm{~km} \mathrm{~s}^{-1}$, $\sigma \approx 4 \mathrm{~km} \mathrm{~s}^{-1}$ is appropriate, but we consider $\sigma=0,2.7$, and $6.8 \mathrm{~km} \mathrm{~s}^{-1}$ so as to examine the dependence on $\sigma$. 11 Many absorption lines of the iodine cell are located between $5000 \AA$ and
$6000 \AA$. 

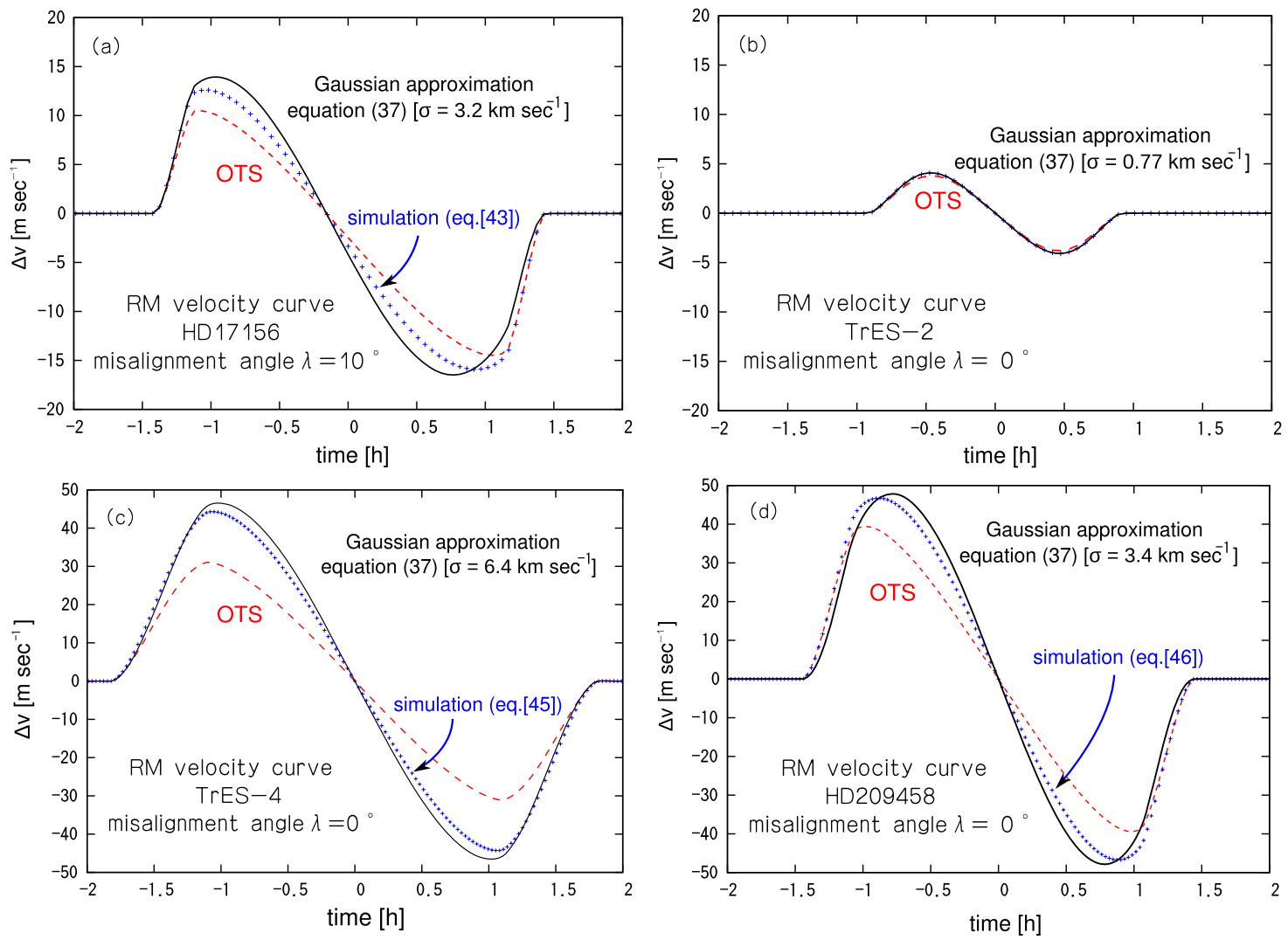

Figure 5. RM velocity anomaly curves for HD 17156 (a), TrES-2 (b), TrES-4 (c), and HD 209458 (d). Crosses indicate the simulated result using the polynomial fit (Equations (43)-(46)). Solid and dashed lines correspond to the Gaussian formula (Equation (37)) and the OTS formula, respectively. The spin-orbit misalignment angle $\lambda$ is assumed to be $10^{\circ}$ for HD 17156 and $0^{\circ}$ other systems. We adopt 87.2, 83.6, 82.6, and 86.7 for the orbital inclination $i_{o}$ of HD 17156, TrES-2, TrES-4, and HD 209458, respectively.

(A color version of this figure is available in the online journal.)

Again we stress here that the lines in the NSO spectrum are not perfectly Gaussian, and the Subaru analysis routine adopts multi-parameters fitting with the least-squares method, instead of the cross-correlation method we assumed in the analytic treatment. Nevertheless, the simulated result and the analytic formula (37) show good overall agreement, indicating the practical validity of our analytic approach.

\subsubsection{Application to Actual Exoplanetary Systems}

While we find that the basic features of the simulated are explained by the Gaussian approximation of both the stellar line profile and the stellar rotation kernel, it still remains to see the extent to which a more realistic rotation kernel affects the RM velocity anomaly. We examine this point by repeating the simulated analysis for HD 17156, TrES-2, TrES-4, and HD 209458 with the rotation kernel (Equation (5)).

1. HD 17156. This transiting planetary system was discovered by Fischer et al. (2007). We assume the linear limbdarkening law with $u_{1}=0.6$ and $u_{2}=0$, and the rotational velocity of $v \sin i_{\star}=4.2 \mathrm{~km} \mathrm{~s}^{-1}$ (Narita et al. 2009). ${ }^{12} \mathrm{We}$ perform the same simulation as before but now using the rotation kernel (Equation (5)), and obtain

$$
\Delta v=-f v_{p}\left[1.37-0.505\left(\frac{v_{p}}{v \sin i_{\star}}\right)^{2}\right] .
$$

12 While Fischer et al. (2007) estimated the stellar rotational velocity to be $v \sin i_{\star}=2.6 \mathrm{~km} \mathrm{~s}^{-1}$, Narita et al. (2009) obtained the stellar rotational velocity $v \sin i_{\star}=4.2 \mathrm{~km} \mathrm{~s}^{-1}$ by the RM analysis. We adopt the latter value here.
The result is plotted in crosses in Figure 5(a). The misalignment angle $\lambda$ is assumed to be $10^{\circ}$. We adopt $i_{o}=87.2$ as the orbital inclination of HD 17156b.

While the rotation kernel is not Gaussian, it is interesting to see the difference between our analytical formula and the simulated result. For this purpose, we fit Equation (5) by a single Gaussian and find the best fit of $\sigma \approx \lambda_{L} / 1.31$ (see Appendix F for detail). The solid line in Figure 5(a) indicates the corresponding result from Equation (37) for $\beta=2.6 \mathrm{~km} \mathrm{~s}^{-1}$ and $\sigma=v \sin i_{\star} / 1.31 \approx 3.2 \mathrm{~km} \mathrm{~s}^{-1}$.

2. TrES-2. The central star in TrES-2 system has a small projected rotational velocity. Winn et al. (2008) investigated the RM effect of this system, and concluded that the orbit of TrES-2b is prograde. They derived the empirical formula for the velocity anomaly using templates based on observations of similar stars. Instead, we use a theoretical G0-type template spectrum without rotational broadening (Coelho et al. 2005), instead of the NSO spectrum, to create the mock spectra. We then broaden the theoretical spectrum assuming $v \sin i_{\star}=1.0 \mathrm{~km} \mathrm{~s}^{-1}$ and the limb-darkening parameters $u_{1}=0.40$ and $u_{2}=0.30$. After inputting the mock spectra into the analysis routine, we obtain the following empirical formula:

$$
\Delta v=-f v_{p}\left[1.06-0.0754\left(\frac{v_{p}}{v \sin i_{\star}}\right)^{2}\right] .
$$

Again, in Figure 5(b), crosses indicate the simulated result (Equation (44)). We also show the OTS formula and the 
Gaussian formula (37), in which $\sigma=v \sin i_{\star} / 1.30=0.766$ $\mathrm{km} \mathrm{s}^{-1}$. We here adopt $1_{o}=83^{\circ} 6$ and $\lambda=0^{\circ}$.

The three different lines are almost indistinguishable due to the small RM amplitude, but we can safely say that the OTS formula as well as the Gaussian formula (37) well describes the simulated result. When the stellar spin velocity is small enough, the OTS formula provides a good approximation to the velocity anomaly.

3. TrES-4. TrES-4 is a transiting planetary system discovered by Mandushev et al. (2007). The parent star is an Ftype star with an effective temperature of $6200 \pm 75 \mathrm{~K}$ and has a relatively high rotation velocity of $v \sin i_{\star}=$ $8.5 \pm 0.5 \mathrm{~km} \mathrm{~s}^{-1}$ (Sozzetti et al. 2009). Since the spectral type of the star is different from that of the Sun, we use theoretically synthesized F-type spectrum. We then broaden the theoretical spectrum assuming a quadratic limb-darkening law (Equation (4)) with $u_{1}=0.46$ and $u_{2}=0.31$ (Claret 2004), and obtain

$$
\Delta v=-f v_{p}\left[1.62-0.885\left(\frac{v_{p}}{v \sin i_{\star}}\right)^{2}\right] .
$$

Figure 5(c) shows the result. The misalignment angle $\lambda$ and the orbital inclination $i_{o}$ are assumed to be $0^{\circ}$ and 82.6, respectively. The solid line in Figure 5 indicates the Gaussian formula with $\sigma=v \sin i_{\star} / 1.32 \approx 6.4 \mathrm{~km} \mathrm{~s}^{-1}$.

4. HD 209458. Finally, we compare the Gaussian formula with the simulated radial velocity anomaly of HD 209458 system. While in Figure 4 the Gaussian broadening kernel is applied to synthesize the mock spectra for stellar rotation, we here adopt the actual rotational kernel (Equation (5)) with $u_{1}=0.45, u_{2}=0.30$, and $v \sin i_{\star}=4.5 \mathrm{~km} \mathrm{~s}^{-1}$. Here is the empirical formula after the mock analysis:

$$
\Delta v=-f v_{p}\left[1.49-0.684\left(\frac{v_{p}}{v \sin i_{\star}}\right)\right] .
$$

As in the above three cases, we show the OTS formula and the Gaussian formula (Equation (37)) in Figure 5(d). We adopt $\sigma=v \sin i_{\star} / 1.31=3.4 \mathrm{~km} \mathrm{~s}^{-1}, i_{o}=86.7$, and $\lambda=0^{\circ}$.

The Gaussian formulae in Figure 5 well describe the behavior of simulated results in each system even though the actual rotation kernels are not described by simple Gaussians. Comparing the three systems except the TrES-2 system, in which the stellar velocity is particularly small, we note that the Gaussian formulae for TrES-4 and HD 209458 describe the simulation better than that for HD 17156. This is presumably due to the fact that apart from the tiny contribution of the tail, the rotation kernel is better approximated as a Gaussian for the star with higher rotational velocity (Appendix F). The fact that the three lines in each figure intersect at the same time (close to, but not identical to, the central transit time $=0$ ) indicates that while the difference among the OTS formula, the Gaussian formula, and the simulated result affects the estimate of $v \sin i_{\star}$, the estimated value of the spin-orbit angle is fairly robust as discussed in Section 2.

We also notice a fairly big discrepancy between the Gaussian formula and the simulated result during the egress of HD 17156 (at $0<$ time $(\mathrm{hr})<1.5$ ) in the upper panel of Figure 5. Indeed, the OTS formula approximates better than the simulated result at the egress phase. In order to understand the behavior, we systematically changed the value of $i_{o}$, and found that the asymmetry of the velocity anomaly curve between an ingress and an egress is very sensitive to the combination of $\lambda$ and $i_{o}$. This points to a limitation of our Gaussian approximation, which might be fixed by using a more precise rotation kernel.

\section{SUMMARY AND DISCUSSION}

We have presented an improved analytic formula for the RM effect as measured from the cross-correlation method. Our main finding is Equation (37), which describes the RM velocity anomaly under the Gaussian approximation for the intrinsic line profile and the stellar rotation kernel. Unlike the previous approximation (the moment method) adopted by OTS, the radial velocity anomaly from the cross-correlation method is explicitly dependent both on the thermal and natural broadening width of an individual line profile $(\beta)$ and on the rotation velocity $(\sigma)$.

The analytical formula has been compared with the simulated analysis for the Subaru HDS routine using the mock spectra constructed from the high-resolution spectrum of the Sun. Even though the actual analysis routine attempts to fit simultaneously many different lines that are not necessarily approximated by Gaussians, the resulting RM velocity anomaly is well described by a form of Equation (37).

The current result explains the previous findings (Winn et al. 2005, 2008; Johnson et al. 2008) that the OTS formula does not provide a good approximation to planetary systems with a relatively high stellar rotation rate. The next step in obtaining a more accurate analytic formula for the RM effect would probably be to use a more accurate rotation kernel (Equation (5)) instead of the Gaussian approximation (Equation (22)). For future work we plan to pursue this approach, together with the propagation of the resulting precision on the misalignment angle $\lambda$ and the rotation velocity $v \sin i_{\star}$.

In addition to the improvement of the analytic approach, the current result points to a possible further refinement of the observational reduction routine for the velocity anomaly due to the RM effect. The procedure described in Section 3.1 is appropriate for the radial velocity determination out of transit. During the transit, however, the Doppler-shifted stellar spectrum $S(\lambda-\delta \lambda)$ in Equation (38) should be replaced with

$$
\begin{aligned}
& S_{\text {star }}(\lambda-\tilde{\lambda})-S_{\text {planet }}(\lambda-\tilde{\lambda}-\Delta \lambda) \\
& \quad=S_{\text {star }}(\lambda-\tilde{\lambda})-f S_{\text {narrowed template }}(\lambda-\tilde{\lambda}-\Delta \lambda),
\end{aligned}
$$

where $S_{\text {star }}(\lambda)$ is the stellar template spectrum derived by the procedure described in Section 3.1 during the out-of-transit data, $S_{\text {narrowed template }}(\lambda)$ is obtained by deconvolving $S_{\text {star }}(\lambda)$ with the rotational kernel in principle, and $\tilde{\lambda}$ and $\Delta \lambda$ correspond to the Keplerian plus peculiar velocity of the star and the radial velocity of the occulted portion of the stellar disk, respectively.

Fitting the observed spectrum in transit with the additional parameters $f, \tilde{\lambda}$, and $\Delta \lambda$, instead of $\delta \lambda$ alone, one may directly extract the velocity $v_{p}=c \Delta \lambda / \lambda_{0}$ after averaged over many different lines. Moreover, one may reduce the number of fitting parameters by estimating $f$ from photometric data during the transit.

Since the above method is more appropriate to model the spectra of the transiting planetary system during a transit, one expects that the precision and accuracy of $\Delta \lambda$, and therefore the RM velocity anomaly, may be improved in principle. In reality, however, the practical feasibility to implement in the analysis 
routine depends on the available resolution of the spectra as well as their stability. The work toward this improvement is also currently under way, which we also hope to present elsewhere in due course.

The RM effect has become an observationally mature probe of the transiting planetary systems. Further improvement in both the accuracy and the precision of the velocity anomaly is important to advance the understanding of the formation and evolution processes of such systems. We hope that the present analytic approach provides some insights that will enable progress in that direction.

We thank Wako Aoki for useful advice on the spectral line profiles. The radial velocity analysis in our simulation was carried out on the computer system at the Astronomy Data Center of the National Astronomical Observatory of Japan. N.N. is supported by a Japan Society for Promotion of Science (JSPS) Fellowship for Research (PD: 20-8141). Y.S. gratefully acknowledges the support from the Global Scholars Program of Princeton University. This work is also supported by JSPS Coreto-Core Program "International Research Network for Dark Energy." J.N.W. gratefully acknowledges the support of the NASA Origins program through award NNX09AD36G.

\section{APPENDIX A}

\section{FLUX DURING A COMPLETE TRANSIT}

In this Appendix, we compute the flux ratio between the flux coming from the stellar disk blocked by the planet and the total flux, and show that Equation (16) reproduces the OTS formula.

In the case of quadratic limb darkening (Equation (4)), we can express $f$ in terms of $\gamma, u_{1}, u_{2}$, and the position of the planet in the stellar disk. The definition of $f$ is

$$
f(x, y) \equiv \frac{\int_{\text {planet }} I\left(x^{\prime}, y^{\prime}\right) d x^{\prime} d y^{\prime}}{\int_{\text {stellar disk }} I\left(x^{\prime}, y^{\prime}\right) d x^{\prime} d y^{\prime}}
$$

where the $x$-and $y$-axes are defined so that the $y$-axis is along the projected rotational axis and the origin is at the center of the stellar disk. The integration in the numerator covers the portion blocked by the planet, while the one in the denominator is performed throughout the whole stellar disk. Denoting the radius of the star by $R_{s}$, then the cosine in Equation (4) is written as

$$
\cos \theta=\sqrt{1-\frac{x^{2}+y^{2}}{R_{s}^{2}}} \equiv \sqrt{1-\frac{r^{2}}{R_{s}^{2}}}
$$

Then the denominator of Equation (A1) becomes

$$
\begin{gathered}
\int_{0}^{R_{S}} d r 2 \pi r\left[1-u_{1}\left\{1-\sqrt{1-\left(\frac{r}{R_{s}}\right)^{2}}\right\}\right. \\
\left.-u_{2}\left\{1-\sqrt{1-\left(\frac{r}{R_{s}}\right)^{2}}\right\}^{2}\right] \\
=\pi R_{s}^{2}\left(1-\frac{1}{3} u_{1}-\frac{1}{6} u_{2}\right) .
\end{gathered}
$$

When the size of the planet is sufficiently small, the numerator of Equation (A1) can be approximated as

$$
\begin{aligned}
\int_{\text {planet }} I\left(x^{\prime}, y^{\prime}\right) d x^{\prime} d y^{\prime} \simeq & I(x, y) \pi R_{s}^{2} \gamma^{2} \\
= & \pi R_{s}^{2} \gamma^{2}\left[1-u_{1}\left\{1-\sqrt{1-\rho^{2}}\right\}\right. \\
& \left.-u_{2}\left\{1-\sqrt{1-\rho^{2}}\right\}^{2}\right]
\end{aligned}
$$

where $\rho \equiv \sqrt{x^{2}+y^{2}} / R_{s}$ and $\gamma=R_{p} / R_{s}$. Substituting Equations (A3) and (A4) into Equation (A1), we obtain

$$
f(x, y) \simeq \frac{1-u_{1}\left\{1-\sqrt{1-\rho^{2}}\right\}-u_{2}\left\{1-\sqrt{1-\rho^{2}}\right\}^{2}}{1-u_{1} / 3-u_{2} / 6} \gamma^{2}
$$

With this expression, Equation (16) reduces to

$$
\Delta v \simeq-v_{p} \frac{\gamma^{2}\left[1-u_{1}\left\{1-\sqrt{1-\rho^{2}}\right\}-u_{2}\left\{1-\sqrt{1-\rho^{2}}\right\}^{2}\right]}{\left(1-u_{1} / 3-u_{2} / 6\right)-\gamma^{2}\left[1-u_{1}\left\{1-\sqrt{1-\rho^{2}}\right\}-u_{2}\left\{1-\sqrt{1-\rho^{2}}\right\}^{2}\right]},
$$

where $v_{p}$ is expressed by the position of the planet as

$$
v_{p}(x, y)=\frac{\iint_{\text {planet }} \Omega_{s}\left(x^{\prime}, y^{\prime}\right) x^{\prime} \sin i_{\star} d x^{\prime} d y^{\prime}}{\iint_{\text {planet }} d x^{\prime} d y^{\prime}} .
$$

The function $\Omega_{s}(x, y)$ is the angular velocity of the stellar spin at position $(x, y)$ and $i$ is the inclination of the stellar spin axis. For rigidly rotating stars, the planet velocity during a complete transit becomes

$$
v_{p}=\Omega_{s} x \sin i_{\star},
$$

with $\Omega_{s}$ being a constant. Equation (A6) with Equation (A8) reproduces the OTS formula for the complete transit with limb darkening (Equation (40) in OTS).

\section{APPENDIX B}

\section{DURING INGRESS AND EGRESS}

During an ingress and an egress of transits, the fraction of the portion on the stellar disk occulted by the planet changes. The center of the stellar disk and the planet is at the origin and at $(x, y)$, respectively.

Figure 6 shows an example of a configuration during an ingress or an egress. We define the radius of the planet as $R_{p}$. The angles $\alpha$ and $\beta$ are given by

$$
\cos \alpha=\frac{R_{s}^{2}+r^{2}-R_{p}^{2}}{2 R_{s} r}, \quad \cos \beta=\frac{R_{p}^{2}+r^{2}-R_{s}^{2}}{2 R_{p} r},
$$

respectively. The size $S$ of the dark shaded area in Figure 6 (the portion occulted by the planet) is

$$
S=\alpha R_{s}^{2}-\frac{1}{2} R_{s}^{2} \sin 2 \alpha+\beta R_{p}^{2}-\frac{1}{2} R_{p}^{2} \sin 2 \beta .
$$

Thus, the fraction $\Gamma(r)$ of the portion on the stellar photosphere occulted by the planet becomes

$$
\Gamma(r) \equiv \frac{S}{\pi R_{s}^{2}}=\frac{1}{\pi}\left\{\alpha-\frac{1}{2} \sin 2 \alpha+\gamma^{2}\left(\beta-\frac{1}{2} \sin 2 \beta\right)\right\} .
$$




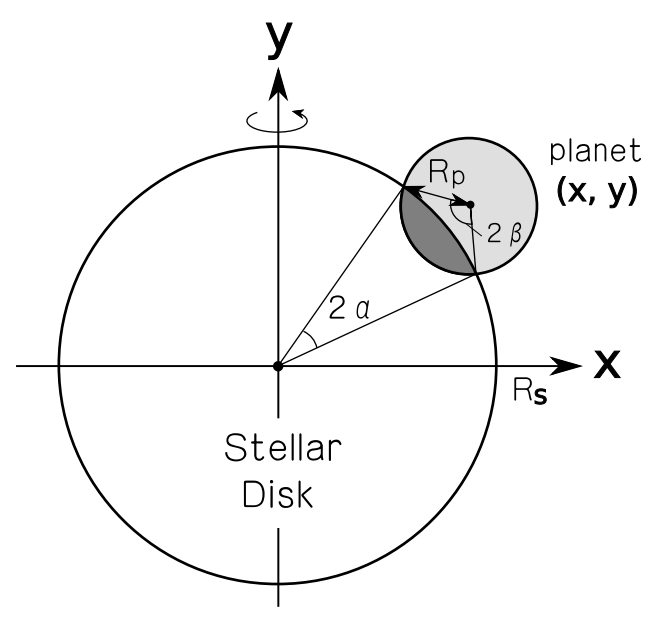

Figure 6. Schematic illustration of the configuration at an ingress or egress. The center of the planet is at $(x, y)$. The dark shaded region is occulted by the planet.

During an ingress or an egress, $\gamma^{2}$ in Equation (A5) must be replaced with $\Gamma(r)$ depending on the position of the planet. This result is derived in the case of $R_{s}<r$, but we can prove that this also holds in the case of $R_{s} \geqslant r$.

The subplanet velocity is no longer expressed by Equation (A8). In this case, we must replace Equation (A8) with

$$
v_{p}(x, y)=\frac{\iint_{S} \Omega_{s} x^{\prime} \sin i_{\star} d x^{\prime} d y^{\prime}}{\iint_{S} d x^{\prime} d y^{\prime}},
$$

where $S$ is the dark shaded region in Figure 6. However, as long as the planet is sufficiently small enough, the approximation (Equation (A8)) provides a good description for subplanet velocity (B4).

\section{APPENDIX C}

\section{DERIVATION OF EQUATION (27)}

We here present the derivation of Equation (27) in detail. Below, we implicitly assume that the quantities $\beta_{\star}$ and $\gamma_{\star}$ represent the broad line originated from the whole photosphere, while $\beta_{p}$ and $\gamma_{p}$ indicate the narrow line originated from the portion of the planet. For further computation, it is convenient to consider the Fourier transforms of the line profile functions. Note that the Fourier transforms of Gaussian and Lorentzian, respectively, become

$$
\begin{gathered}
G(\lambda)=\frac{1}{\beta \sqrt{\pi}} e^{-\lambda^{2} / \beta^{2}} \Longrightarrow \tilde{G}(\sigma)=e^{-\pi^{2} \beta^{2} \sigma^{2}}, \\
L(\lambda)=\frac{1}{\pi} \frac{\gamma^{2}}{\lambda^{2}+\gamma^{2}} \Longrightarrow \tilde{L}(\sigma)=e^{-2 \pi \gamma|\sigma|} .
\end{gathered}
$$

Then, the Fourier transform of the Voigt function becomes $\tilde{V}(\sigma)=\tilde{G}(\sigma) \tilde{L}(\sigma)$. In terms of these, Equations (25) and (26) are rewritten as

$$
\mathcal{F}_{\text {transit }}(\lambda)=-\int_{-\infty}^{\infty} d \sigma\left[\tilde{V}_{\star}(\sigma) e^{-2 \pi i \sigma \lambda}-f \tilde{V}_{p}(\sigma) e^{-2 \pi i \sigma(\lambda-\Delta \lambda)}\right],
$$

$$
\mathcal{F}_{\text {star }}(\lambda)=-\int_{-\infty}^{\infty} d \sigma \tilde{V}_{\star}(\sigma) e^{-2 \pi i \sigma(\lambda)},
$$

where $\tilde{V}_{\star}(\sigma)$ and $\tilde{V}_{p}(\sigma)$ are the Fourier transforms of $G\left(\lambda ; \beta_{\star}\right) L\left(\lambda ; \gamma_{\star}\right)$ and $G\left(\lambda ; \beta_{p}\right) L\left(\lambda ; \gamma_{p}\right)$, respectively. The corresponding Fourier counterpart are

$$
\begin{gathered}
\tilde{\mathcal{F}}_{\text {transit }}(\sigma)=-\tilde{V}_{\star}(\sigma)+f \tilde{V}_{p}(\sigma) e^{2 \pi i \sigma \Delta \lambda}, \\
\tilde{\mathcal{F}}_{\text {star }}(\sigma)=-\tilde{V}_{\star}(\sigma) .
\end{gathered}
$$

Thus, we obtain

$$
C\left(\delta_{\mathrm{RM}}\right)=\int_{-\infty}^{\infty} d \sigma \tilde{C}(\sigma) e^{2 \pi i \sigma \delta_{\mathrm{RM}}},
$$

where

$$
\begin{aligned}
\tilde{C}(\sigma) & =\tilde{\mathcal{F}}_{\text {star }}(\sigma) \times \tilde{\mathcal{F}}_{\text {transit }}^{*}(\sigma) \\
& =\tilde{V}_{\star}(\sigma)\left(\tilde{V}_{\star}(\sigma)-f \tilde{V}_{p}(\sigma) e^{-2 \pi i \sigma \Delta \lambda}\right) .
\end{aligned}
$$

Now it is easy to incorporate additional effects expressed as a convolution simply by multiplying the Fourier transform of the additional convolution kernel.

Substituting Equations (C1) and (C2) into Equation (C8), we obtain

$$
\begin{aligned}
C(\sigma)= & \int_{-\infty}^{\infty} d \sigma \exp \left[-2 \pi^{2} \beta_{\star}^{2} \sigma^{2}-4 \pi \gamma_{\star}|\sigma|+2 \pi i \sigma \delta_{\mathrm{RM}}\right] \\
& -f \int_{-\infty}^{\infty} d \sigma \exp \left[-\pi^{2}\left(\beta_{\star}^{2}+\beta_{p}^{2}\right) \sigma^{2}-2 \pi\left(\gamma_{\star}+\gamma_{p}\right)|\sigma|\right. \\
& \left.+2 \pi i \sigma\left(\delta_{\mathrm{RM}}-\Delta \lambda\right)\right] .
\end{aligned}
$$

The integral of each term in Equation (C9) results in the following integral:

$$
\xi(a, b, c) \equiv \int_{0}^{\infty} \exp \left(-b x^{2}+c x\right) \cos (a x) d x
$$

The derivative of the function $\xi(a, b, c)$ with respect to $a$ is

$$
\begin{aligned}
& \frac{\partial}{\partial a} \xi(a, b, c)=-\frac{1}{8 b^{3 / 2}} e^{-\left(a^{2}-c^{2}\right) / 4 b} \\
& \times \sqrt{\pi}\left[(a-i c) e^{i a c / 2 b}\left(1+\operatorname{erfi}\left(\frac{a-i c}{2 \sqrt{b}}\right)\right)+\text { c.c. }\right] .
\end{aligned}
$$

In terms of $\xi(a, b, c)$, Equation (C9) is expressed as

$$
\begin{aligned}
C(\sigma)= & \xi\left(2 \pi \delta_{\mathrm{RM}}, 2 \pi^{2} \beta_{\star}^{2}, 4 \pi \gamma_{\star}\right) \\
& -f \xi\left(2 \pi\left(\delta_{\mathrm{RM}}-\Delta \lambda\right), \pi^{2}\left(\beta_{\star}^{2}+\beta_{p}^{2}\right), 2 \pi\left(\gamma_{\star}+\gamma_{p}\right)\right) .
\end{aligned}
$$

Substituting Equation (C12) into Equation (17) and using Equation (C11), we finally obtain an equation for $\delta_{\mathrm{RM}}$ :

$$
\begin{aligned}
\frac{1}{b^{3 / 2}} & e^{-\left(a^{2}-c^{2}\right) / 4 b} \operatorname{Re}\left[(a-i c) e^{i a c / 2 b}\left(1+\operatorname{erfi}\left(\frac{a-i c}{2 \sqrt{b}}\right)\right)\right] \\
= & \frac{f}{B^{3 / 2}} e^{-\left(A^{2}-C^{2}\right) / 4 B} \operatorname{Re}\left[(A-i C) e^{i A C / 2 B}\right. \\
& \left.\times\left(1+\operatorname{erfi}\left(\frac{A-i C}{2 \sqrt{B}}\right)\right)\right]
\end{aligned}
$$


where

$$
\begin{gathered}
a=2 \pi \delta_{\mathrm{RM}}, b=2 \pi^{2} \beta_{\star}^{2}, c=4 \pi \gamma_{\star}, \\
A=2 \pi\left(\delta_{\mathrm{RM}}-\Delta \lambda\right), B=\pi^{2}\left(\beta_{\star}^{2}+\beta_{p}^{2}\right), C=2 \pi\left(\gamma_{\star}+\gamma_{p}\right) .
\end{gathered}
$$

\section{APPENDIX D}

\section{LORENTZIAN PROFILE}

In Section 2.3, we have derived an analytic expression for Gaussian line profiles. In this Appendix, we consider the opposite simplification that the line profile has a Lorentzian form $(b=B=0)$. To derive the radial velocity formula, we use

$$
\begin{aligned}
\lim _{b \rightarrow 0} & \frac{1}{b^{3 / 2}} e^{-\left(a^{2}-c^{2}\right) / 4 b} \operatorname{Re}\left[(a-i c) e^{i a c / 2 b}\left(1+\operatorname{erfi}\left(\frac{a-i c}{2 \sqrt{b}}\right)\right)\right] \\
& =-\frac{8}{\sqrt{\pi}} \frac{a c}{\left(a^{2}+c^{2}\right)^{2}} .
\end{aligned}
$$

Then, Equation (27) or Equation (C13) reduces to

$$
\frac{2 \delta_{\mathrm{RM}} \gamma_{\star}}{\left(\delta_{\mathrm{RM}}^{2}+4 \gamma_{\star}^{2}\right)}=f \frac{\left(\delta_{\mathrm{RM}}-\Delta \lambda\right)\left(\gamma_{\star}+\gamma_{p}\right)}{\left\{\left(\delta_{\mathrm{RM}}-\Delta \lambda\right)^{2}+\left(\gamma_{\star}+\gamma_{p}\right)^{2}\right\}^{2}} .
$$

Employing the similar approximation in Section 2.3.1, we obtain an approximate expression for the Lorentzian profile:

$$
\delta_{\mathrm{RM}} \simeq-\left(\frac{2 \gamma_{\star}}{\gamma_{\star}+\gamma_{p}}\right)^{3} f \Delta \lambda\left\{1-2\left(\frac{\Delta \lambda}{\gamma_{\star}+\gamma_{p}}\right)^{2}\right\},
$$

or

$$
\Delta v \simeq-\left(\frac{2 \gamma_{\star}}{\gamma_{\star}+\gamma_{p}}\right)^{3} f v_{p}\left\{1-\frac{2 \lambda_{0}^{2}}{c^{2}\left(\gamma_{\star}+\gamma_{p}\right)^{2}} v_{p}^{2}\right\} .
$$

Again, we obtain the similar expression for Equation (37). Note that the factor in front of $f$ depends on the widths of Lorentzians, which generally differs from unity. The presence of the cubic term in $v_{p}^{3}$ is also a major difference from the result by the moment method.

\section{APPENDIX E}

\section{VELOCITY ANOMALY FOR THE GAUSSIAN PROFILE PERTURBED WITH LORENTZIAN}

We discuss a more general case in which the line profile is expressed as a Gaussian profile with a small contribution from the Lorentzian profile. We assume that $\gamma / \beta$ is sufficiently small (the Gaussian property is dominant), and collect the terms up to the linear order of $\gamma / \beta$ in Equation (27). Noting that

$$
\operatorname{erfi}(z)=\frac{2 z}{\sqrt{\pi}} z \ll 1
$$

and defining $z=(a-i c) / 2 \sqrt{b}$, the right-hand side of Equation (27) becomes

$$
\begin{aligned}
\frac{2}{b} \operatorname{Re}\left[z e^{-z^{2}}\left(1+\frac{2 i}{\sqrt{\pi}} z\right)\right] & \simeq \frac{2}{b} \operatorname{Re}\left[z\left(1-z^{2}\right)\left(1+\frac{2 i}{\sqrt{\pi}} z\right)\right] \\
& \simeq \frac{2 a}{b \sqrt{b}}\left(\frac{1}{2}+\frac{c}{\sqrt{\pi b}}-\frac{a^{2}-3 c^{2}}{8 b}\right) .
\end{aligned}
$$

Expanding the left-hand side of Equation (27) in a similar way, and substituting $a, b, c$ and $A, B, C$, we obtain

$$
\begin{aligned}
\delta_{\mathrm{RM}} \simeq & -\left(\frac{2 \beta_{\star}^{2}}{\beta_{\star}^{2}+\beta_{p}^{2}}\right)^{3 / 2} \frac{f \Delta \lambda}{1+\frac{8 \gamma_{\star}}{\sqrt{2 \pi \beta_{\star}^{2}}}} \\
& \times\left\{1+\frac{4\left(\gamma_{\star}+\gamma_{p}\right)}{\sqrt{\pi\left(\beta_{\star}^{2}+\beta_{p}^{2}\right)}}-\frac{\Delta \lambda^{2}}{\beta_{\star}^{2}+\beta_{p}^{2}}\right\},
\end{aligned}
$$

or

$$
\begin{aligned}
\Delta v \simeq & -\left(\frac{2 \beta_{\star}^{2}}{\beta_{\star}^{2}+\beta_{p}^{2}}\right)^{3 / 2} \frac{1}{1+\frac{8 \gamma_{\star}}{\sqrt{2 \pi \beta_{\star}^{2}}}} f v_{p} \\
& \times\left\{1+\frac{4\left(\gamma_{\star}+\gamma_{p}\right)}{\sqrt{\pi\left(\beta_{\star}^{2}+\beta_{p}^{2}\right)}}-\frac{\lambda^{2}}{c^{2}\left(\beta_{\star}^{2}+\beta_{p}^{2}\right)} v_{p}^{2}\right\} .
\end{aligned}
$$

This is the formula for the velocity anomaly when absorption lines are Gaussians perturbed with the Lorentzian profile. Note that Equation (E4) reproduces Equation (37) when we set $\gamma_{\star}=\gamma_{p}=0$.

\section{APPENDIX F}

\section{RELATION BETWEEN THE ROTATION AND GAUSSIAN BROADENING KERNELS BY LEAST-SQUARES FITTING}

Here, we derive a scaling factor between the upper-limit wavelength $\lambda_{L}$ of the rotational kernel and the dispersion $\sigma$ of the Gaussian broadening kernel by least-squares fitting. Although replacing a rotational kernel with a Gaussian one is a crude approximation, we can justify this treatment to some extent (Section 3.3.2.).

We define $\Delta^{2}$ as the residual between the two convolution kernels:

$$
\begin{aligned}
\Delta^{2}= & \int_{-\infty}^{\infty}\left[R\left(\lambda ; \lambda_{L}\right)-G(\lambda ; \beta)\right]^{2} d \lambda \\
= & \int_{-\infty}^{\infty} R^{2}\left(\lambda ; \lambda_{L}\right) d \lambda+\int_{-\infty}^{\infty} G^{2}(\lambda ; \beta) d \lambda \\
& -2 \int_{-\infty}^{\infty} R\left(\lambda ; \lambda_{L}\right) G(\lambda ; \beta) d \lambda .
\end{aligned}
$$

The scaling factor $\alpha$ is defined as

$$
\alpha \equiv \frac{\lambda_{L}}{\beta} .
$$

When we fix the rotational velocity (thus $\lambda_{L}$ ), $\alpha$ is determined by minimizing of the function $\Delta^{2}$ :

$$
\frac{\partial \Delta^{2}}{\partial \alpha}=0
$$

Since the first term in Equation (F1) does not depend on $\beta$, we neglect it in computing the derivative in terms of $\beta$. The second term is written as

$$
\int_{-\infty}^{\infty} G^{2}(\lambda ; \beta) d \lambda=\frac{1}{\beta \sqrt{2 \pi}}=\frac{\alpha}{\lambda_{L} \sqrt{2 \pi}} .
$$


The third term in Equation (F1) reduces to

$$
\begin{aligned}
\int_{-\infty}^{\infty} & R\left(\lambda ; \lambda_{L}\right) G(\lambda ; \beta) d \lambda=\frac{\lambda_{L}}{\beta \sqrt{\pi}}\left[c_{1} \int_{-1}^{1} e^{-\alpha^{2} x^{2}} \sqrt{1-x^{2}} d x\right. \\
& \left.+c_{2} \int_{-1}^{1} e^{-\alpha^{2} x^{2}}\left(1-x^{2}\right) d x+c_{3} \int_{-1}^{1} e^{-\alpha^{2} x^{2}}\left(1-x^{2}\right)^{3 / 2} d x\right] \\
= & \frac{\alpha}{\sqrt{\pi}}\left[c_{1} \frac{\pi}{2} e^{-\alpha^{2} / 2}\left\{I_{0}\left(\alpha^{2} / 2\right)+I_{1}\left(\alpha^{2} / 2\right)\right\}\right. \\
& +c_{2} \frac{1}{2 \alpha^{3}}\left\{2 \alpha e^{-\alpha^{2}}+\left(-1+2 \alpha^{2}\right) \sqrt{\pi} \operatorname{erf}(a)\right\} \\
& \left.+c_{3} \frac{\pi}{2 \alpha^{2}} e^{-\alpha^{2} / 2}\left\{\alpha^{2} I_{0}\left(\alpha^{2} / 2\right)+\left(-1+\alpha^{2}\right) I_{1}\left(\alpha^{2} / 2\right)\right\}\right], \quad(\mathrm{F} 5)
\end{aligned}
$$

where $I_{n}(x)$ is the $n$ th-order modified Bessel function. Substituting Equations (6)-(8) into Equation (F5), we obtain the derivative of $\Delta^{2}$ :

$$
\begin{aligned}
\frac{\partial \Delta^{2}}{\partial \alpha}= & \frac{1}{\lambda_{L} \sqrt{\pi}}\left[\frac{1}{\sqrt{2}}-\frac{12\left(u_{1}+2 u_{2}\right) e^{-\alpha^{2}}}{\alpha^{2}\left(-6+2 u_{1}+u_{2}\right)}\right. \\
& +\frac{6 e^{-\alpha^{2} / 2}}{\alpha^{3}\left(-6+2 u_{1}+u_{2}\right)}\left\{-2 \alpha^{3}\left(-1+u_{1}+u_{2}\right) I_{0}\left(\alpha^{2} / 2\right)\right. \\
& +2 \alpha\left\{-2 u_{2}+\alpha^{2}\left(-1+u_{1}+u_{2}\right)\right\} I_{1}\left(\alpha^{2} / 2\right) \\
& \left.\left.+\sqrt{\pi} e^{\alpha^{2} / 2}\left(u_{1}+2 u_{2}\right) \operatorname{erf}(\alpha)\right\}\right]=0 .
\end{aligned}
$$

Substituting the limb-darkening parameters, we numerically solve the above equation for $\alpha$. We obtain $\alpha \simeq 1.31$ for HD $17156\left(u_{1}=0.6, u_{2}=0\right), \alpha \simeq 1.30$ for TrES-2 $\left(u_{1}=0.40\right.$, $\left.u_{2}=0.30\right), \alpha \simeq 1.32$ for TrES-4 $\left(u_{1}=0.46, u_{2}=0.31\right)$, and $\alpha \simeq 1.31$ for HD $209458\left(u_{1}=0.45, u_{2}=0.30\right)$. Equation (F6) implies that residuals $\Delta^{2}$ scale as $\lambda_{L}^{-1}$ when we fix $\alpha$. This means that for a rapidly rotating star, $\Delta^{2}$ becomes small and the approximation of a rotational kernel with Gaussian is validated.

\section{REFERENCES}

Butler, R. P., Marcy, G. W., Williams, E., McCarthy, C., Dosanjh, P., \& Vogt, S. S. 1996, PASP, 108, 500

Chatterjee, A., Sarkar, A., Barat, P., Mukherjee, P., \& Gayathri, N. 2008, ApJ, 686,580

Claret, A. 2004, A\&A, 428, 1001

Coelho, P., Barbuy, B., Meléndez, J., Schiavon, R. P., \& Castilho, B. V. 2005, A\&A, 443, 735

Fischer, D. A., et al. 2007, ApJ, 669, 1336

Gaudi, B. S., \& Winn, J. N. 2007, ApJ, 655, 550

Gray, D. F. 2005, The Observation and Analysis of Stellar Photospheres (3rd ed.; Cambridge: Cambridge Univ. Press)

Ida, S., \& Lin, D. N. C. 2004, ApJ, 616, 567

Johnson, J. A., et al. 2008, ApJ, 686, 649

Kopal, Z. 1942, Proc. Natl. Acad. Sci. USA, 28, 133

Kurucz, R. L., Furenlid, I., Brault, J., \& Tsterman, L., ed. 1984, in Solar Flux Atlas from 296 to $1300 \mathrm{~nm}$ (New Mexico: National Solar Observatory)

Lin, D. N. C., Bodenheimer, P., \& Richardson, D. C. 1996, Nature, 380, 606

Mandushev, G., et al. 2007, ApJ, 667, L195

McLaughlin, D. B. 1924, ApJ, 60, 22

Nagasawa, M., Ida, S., \& Bessho, T. 2008, ApJ, 678, 498

Narita, N., Sato, B., Ohshima, O., \& Winn, J. N. 2008, PASJ, 60, L1

Narita, N., et al. 2007, PASJ, 59, 763

Narita, N., et al. 2009, PASJ, 61, 991

Ohta, Y., Taruya, A., \& Suto, Y. 2005, ApJ, 622, 1118 (OTS)

Ohta, Y., Taruya, A., \& Suto, Y. 2009, ApJ, 690, 1

Queloz, D., Eggenberger, A., Mayor, M., Perrier, C., Beuzit, J. L., Naef, D., Sivan, J. P., \& Udry, S. 2000, A\&A, 359, L13

Rossiter, R. A. 1924, ApJ, 60, 15

Sato, B., Kambe, E., Takeda, Y., Izumiura, H., \& Ando, H. 2002, PASJ, 54, 873

Sozzetti, A., et al. 2009, ApJ, 691, 1145

Triaud, A. H. M. J., et al. 2009, A\&A, 506, 377

Winn, J. N., et al. 2005, ApJ, 631, 1215

Winn, J. N., et al. 2007, ApJ, 665, L167

Winn, J. N., et al. 2008, ApJ, 682, 1283

Wu, Y., Murray, N. W., \& Ramsahai, J. M. 2007, ApJ, 670, 820 